\title{
How Do We Move Forward with Vaccines in Lung Cancer?
}

\author{
Anouck Ottevaere, ${ }^{1}$ Nele De Brucker ${ }^{1}$ and Johan Vansteenkiste ${ }^{2}$ \\ 1. Consultant; 2. Professor of Internal Medicine, Respiratory Oncology Unit (Department of Respiratory Diseases) and Leuven Lung Cancer Group, \\ University Hospital KU Leuven, Leuven, Belgium
}

\begin{abstract}
Vaccination for non-small cell lung cancer has been studied in several large phase III trials over the past decade. They confirmed excellent tolerability, but could not demonstrate outcome benefits, despite promising earlier phase II randomised studies. MAGE-A3 as Adjuvant Non-Small Cell LunG CanceR ImmunoTherapy (MAGRIT), the largest therapeutic trial ever carried out in lung cancer, with appropriate setting, power and design, answered a long-existing question: therapeutic vaccination with current technologies does not work in lung cancer. The best strategy forward may be combination of neo-epitopes vaccines and checkpoint inhibitors.
\end{abstract}

\section{Keywords}

Non-small cell lung cancer, cancer vaccines, immunotherapy

\begin{abstract}
Disclosure: Anouck Ottevaere and Nele De Brucker have no conflicts of interest to declare. Johan Vansteenkiste has advisory functions for Boehringer-Ingelheim, BMS, MSD, Novartis and Eli-Lilly. No funding was received in the publication of this article.

open Access: This article is published under the Creative Commons Attribution Noncommercial License, which permits any non-commercial use, distribution, adaptation and reproduction provided the original author(s) and source are given appropriate credit. DOI: http://doi.org/10.17925/EOH.2015.11.02.92

Received: 2 July 2015 Published Online: 16 November 2015 Citation: European Oncology \& Haematology, 2015;11(2):92-3

Correspondence: Johan Vansteenkiste, Respiratory Oncology Unit (Department of Respiratory Diseases), Herestraat 49, 3000 Leuven, Belgium.

E: johan.vansteenkiste@uzleuven.be
\end{abstract}

Over the last decade, targeted agents have clearly improved treatment options for molecularly selected patients with advanced non-small cell lung cancer (NSCLC) harbouring an oncogene driver, such as an activating epidermal growth factor receptor (EGFR) gene mutation or an anaplastic lymphoma kinase (ALK) gene translocation. ${ }^{1,2}$ But for the majority of Caucasian patients, standard chemotherapy remains the preferred choice of treatment, in locally advanced stages, ${ }^{3}$ and as primary treatment in metastatic disease, as only 15 to $20 \%$ of patients have a treatable driver oncogene. ${ }^{4}$ So there is room left for improvement of therapy.

Nowadays, immunotherapy becomes increasingly important in the treatment of NSCLC. There are two main approaches: one is antigenspecific immunotherapy or therapeutic cancer vaccination, the other is non-antigen-specific immunotherapy or immunomodulation, recently delivering great results with so-called immune checkpoint inhibitors. These agents are monoclonal antibodies that block the programmed death 1 (PD-1) receptor on the lymphocytes or its ligand (PD-L1) on tumour cells, in order to restore anti-tumour immunity. In recently presented results, the anti-PD-1 antibody nivolumab was clearly superior to docetaxel for relapse therapy of squamous NSCLC. ${ }^{5}$

The aim of vaccination is to potentiate the immune response against specific tumour-associated antigens, by adding an adjuvant (viral vector, a dendritic cell presentation, a phospholipid, a liposomal presentation or an aluminium formulation) to one or several tumourassociated antigens (peptides, recombinant full proteins, gangliosides or whole tumour cells). Over the last decade, results of several large clinical trials have been reported.
In 2013, the results of the phase III tecemotide (L-BLP25) trial (Stimulating Targeted Antigenic Responses To NSCLC [START]) were presented at the American Society of Clinical Oncology (ASCO) meeting and subsequently published. ${ }^{\text {This }}$ vaccine targets MUC1 protein. MUC1 is found on the surface of many epithelial cells, but in carcinoma cells MUC1 is overexpressed and has an aberrant glycosylation, thereby exposing the protein core as a target for the immune system. Tecemotide is based on repeat 25 amino acid peptides of this core protein. In this phase III double-blind study, patients with unresectable stage III NSCLC, who did not progress after sequential or concurrent chemoradiotherapy, were randomly assigned to maintenance therapy with the vaccine versus placebo. There was no significant difference in overall survival (OS) in the whole trial, but a pre-planned subgroup analysis (still on $>800$ patients) revealed a 10-month improvement in median OS (30.8 versus 20.6 months) in patients who received concurrent chemoradiotherapy, in contrast with those treated with sequential chemoradiotherapy. The consequential phase III trial (START2), in patients with stage III NSCLC treated with concurrent chemoradiotherapy, was unfortunately abandoned because of disappointing results of a smaller Japanese trial in a similar setting.?

In 2013 as well, the results of the belagenpumatucel-L vaccine were presented. ${ }^{8}$ This vaccine is based on whole tumour cells, derived from four irradiated NSCLC cell lines (one large cell, one squamous and two adenocarcinoma), which exhibit transforming growth factor-beta 2 (TGF- $\beta 2$ ) gene knockdown as adjuvant principle. TGF- $\beta 2$ is indeed a major factor in the immunosuppressive environment of NSCLC. The phase III STOP trial included patients with stage III and IV NSCLC with controlled disease after first-line therapy. Patients were randomly assigned to receive the vaccine monthly during 18 months and at 21 and 24 months, or placebo. Again, OS did not differ significantly in the 
overall population, but in subgroup analyses, there was a significant benefit in median OS in patients who received prior radiation therapy (40.1 versus 10.3 months). However, because of the very low numbers in these subgroups, the meaning of these data is questionable.

In 2014, the results of the phase III MAGE-A3 as Adjuvant Non-Small Cell LunG CanceR ImmunoTherapy (MAGRIT) trial were presented. ${ }^{9}$ This vaccine focuses on MAGE-A3, which is a protein that is not expressed in normal tissue, but appears in $35 \%$ of early and up to $55 \%$ of advanced stages of NSCLC. ${ }^{10}$ The MAGE-A3 vaccine consists of a recombinant fusion protein (MAGE-A3 and protein D of Haemophilus influenzae) and a proprietary adjuvant system (AS02B). The MAGE-A3 vaccine is delivered for five administrations every 3 weeks, followed by eight administrations every 3 months. A proof-of-principle phase II randomised placebo-controlled study - conducted in MAGE-A3positive patients with completely resected stage IB/II NSCLC - had shown a promising hazard ratio in disease-free survival. ${ }^{11}$ Therefore, the large, worldwide, multicentre, double-blind phase III MAGRIT trial was developed. In this trial, MAGE-A3-positive patients with completely resected stage $\mathrm{IB}$, II or IIIA NSCLC were randomised 2:1 to receive MAGE-A3 vaccine or placebo, after they also had received adjuvant chemotherapy as clinically indicated. Almost 14,000 surgical patients were screened, 4,210 tumours had MAGE-A3 expression and 2,312 patients were randomised. Unfortunately, the median disease-free survival in the overall population showed no significant difference, being 60.5 months for MAGE-A3, and 57.9 months for placebo (hazard ratio [HR] 1.02; $95 \%$ confidence interval [CI] 0.89-1.18). No benefit was observed in any subset analysis. So, despite appropriate setting (minimal disease post-surgery), design and power of this trial, results were disappointing.

Another vaccine, TG4010, is still in phase III testing. It also targets MUC1, but, in contrast with tecemotide, the antigen is the full MUC1 protein. The adjuvant is a recombinant viral vector expressing the tumour-associated antigen MUC1 and interleukin-2. In the underlying phase II randomised study, patients with MUC1 positive non-radically treatable stage IIIB or IV NSCLC were randomly assigned to cisplatin-gemcitabine chemotherapy up to six cycles, with or without the TG4010 vaccine.12 There was no significant difference in OS, but progression-free survival (PFS) at 6 months in the vaccinated group (43.2 \%) met the pre-defined primary endpoint of a PFS above $40 \%$. The phase IIB/III TIME study now further explores the possible role of TG4010 vaccination in combination with standard first-line chemotherapy in MUC1-positive stage IV NSCLC (NCT01383148).

In conclusion, antigen-specific immunotherapy in NSCLC has been studied in several well-designed and sufficiently powered studies, with overall disappointing results. All of these trials have confirmed that vaccination therapy is very well tolerated, but could not demonstrate clinically useful benefits. The phase III trial with tecemotide showed interesting data, with a 10-month improvement in median OS after concurrent chemoradiotherapy for stage III NSCLC, but unfortunately further investigation was stopped. The MAGRIT trial, the largest therapeutic trial ever carried out in lung cancer, had an appropriate setting, power and design. The merit of this trial thus is that it answers a long-existing question: therapeutic vaccination with current technologies does not work in lung cancer.

Therefore better understanding of mechanisms of failure is needed. We know that vaccination leads to a clear immune response (e.g. MAGE-A3 vaccination did result in antigen-specific antibodies and cytotoxic cells), but apparently this response is not sufficient to result in clinically relevant progress. The most plausible hypothesis is that the immune effectors generated by vaccination and demonstrated in the circulation, are not able to act once they come in the immunosuppressive micro-environment of the tumour. Therefore, the best strategy forward may be the combination of vaccines with neo-epitopes and checkpoint inhibitors, as the latter reverse this immunosuppressive environment.
1. Mok TS, WU YL, Thongprasert S, et al., Gefitinib or carboplatinpaclitaxel in pulmonary adenocarcinoma. N Eng/ I Med, paclitaxel in pulmon

2. Shaw AT, Kim DW, Mehra R, et al., Ceritinib in ALKrearranged non-small cell lung cancer, N Eng/ J Med, 2014;370:1189-97.

3. Vansteenkiste J, De Ruysscher D, Eberhardt W, et al., Early and locally advanced non-small cell lung cancer (NSCLC): ESMO Clinical Practice Guidelines for diagnosis, treatment and follow-up, Ann Oncol, 2013;24(Suppl. 6):vi89-vi98.

4. Reck M, Popat S, Reinmuth $\mathrm{N}$, et al., Metastatic non-smallcell lung cancer (NSCLC): ESMO Clinical Practice Guidelines for diagnosis, treatment and follow-up, Ann Oncol, 2014;25(Suppl. 3):iii27-iii39.

5. Brahmer J, Reckamp KL, Baas P, et al., Nivolumab versus docetaxel in advanced squamous cell non-small cell lung cancer, N Eng/ J Med, 2015 [Epub ahead of print].

6. Butts C, Socinski MA, Mitchell PL, et al., Tecemotide (L-BLP25) versus placebo after chemoradiotherapy for stage III nonsmall-cell lung cancer (START): A randomised, double-blind, phase 3 trial, Lancet Oncol, 2014;15:59-68.

7. Tecemotide clinical development programme discontinuation. Available at: http://www.merckgroup.com/en/media/ extNewsDetail.html?newsId=8475BA17A3F51470C1257D5000 6901B4\&newsType $=1$ (accessed 2 September 2015)

8. Giaccone G, Bazhenova L, Nemunaitis J, et al., A phase III study of belagenpumatucel-L therapeutic tumor cell vaccine for non-small cell lung cancer (NSCLC), Eur J Cancer, 2013;47(Suppl. 2):abstract LBA 7081

9. Vansteenkiste JF, Cho BC, Vanakesa T, et al., MAGRIT, a double-blind, randomized, placebo-controlled phase III study to assess the efficacy of the recMAGE-A3 + AS15 cancer immunotherapeutic as adjuvant therapy in patients with resected MAGE-A3-positive non-small cell lung cancer (NSCLC), Ann Oncol, 2014;25(Suppl. 4):Abstract 11730.

10. Sienel W, Varwerk $C$, Linder $A$, et al., Melanoma associated antigen (MAGE)-A3 expression in stages I and II non-small cell lung cancer: results of a multi-center study, Eur J Cardiothorac Surg, 2004;25:131-4.

11. Vansteenkiste J, Zielinski $M$, Linder $A$, et al., Adjuvant MAGE-A3 immunotherapy in resected non-small cell lung cancer: Phase II randomized study results, J Clin Oncol, 2013;31:2396-403

12. Quoix E, Ramlau R, Westeel V et al., Therapeutic vaccination with TG4010 and first-line chemotherapy in advanced nonsmall cell lung cancer: A controlled phase $2 \mathrm{~B}$ trial, Lancet Oncol, 2011;12:1125-33. 